\title{
TAGUNG
}

\section{Zuhören statt entscheiden: Die Rolle sozialer Medien in der europapolitischen Kommunikation}

\author{
Julian Plottka*
}

Die Nutzung sozialer Medien ist nicht erst durch die Piraten-Partei in die politische Kommunikation eingeführt worden. Auch etablierte politische Akteure bedienen sich, teils länger als es die Piraten gibt, sozialer Medien in ihrer politischen Kommunikation. Dennoch sind die jüngsten Wahlerfolge der Piraten-Partei Anzeichen dafür, dass viele Fragen zur Kompatibilität sozialer Medien mit den bestehenden Systemen repräsentativer Demokratie zum Teil noch nicht ausreichend debattiert werden und zum Teil noch nicht einmal formuliert sind.

Die europapolitische Kommunikation in den Blick nehmend wurden auf der Tagung sowohl aus wissenschaftlicher Sicht wie aus Perspektive von Praktikern des politischen Geschäfts einige dieser Fragen aufgeworfen und diskutiert: Was ist unter sozialen Medien und dem schillernden Begriff des Web 2.0 überhaupt zu verstehen? Wie verändern soziale Medien das Kommunikationsverhalten von Menschen? Worin liegen die Chancen und Risiken sozialer Medien für die politische Kommunikation? Welche Herausforderungen stellen soziale Medien für Bürger und Politiker dar? Verändern soziale Medien die repräsentative Demokratie, insbesondere dadurch, dass sie mehr Partizipation der Bürger ermöglichen? ${ }^{1}$

Dass keine abschließenden Ergebnisse präsentiert werden konnten, ergibt sich aus beiden

Social Media in der Europapolitischen
Kommunikation
Gemeinsame Tagung des Europa Zentrums Baden-
Württemberg, der MFG Innovationsagentur für IT
und Medien, Baden-Württemberg, des Arbeitskrei-
ses Europäische Integration und der Alcatel-Lucent
$\quad$ Stiftung für Kommunikationsforschung
$\quad$ Medienpartner SWRinfo
25.-26. Juni 2012, Stuttgart
Mit Unterstützung der Europäischen Kommission
Tagungsleitung/Moderation
Florian SETZEN, Direktor, Europa Zentrum Ba-
den-Württemberg, Stuttgart
Ulrich WINCHENBACH, Leiter Projektteam Wei-
terbildung/Events, MFG Innovationsagentur für IT
und Medien Baden-Württemberg, Stuttgart
Begrüßung
Helena ACHESON, Unitleiterin Innovationspro-
jekte/Business Development/Communications,
MFG Innovationsagentur für IT und Medien Ba-
den-Württemberg, Stuttgart
Florian H. SETZEN, Direktor, Europa Zentrum Ba-
den-Württemberg, Stuttgart
Das Verhältnis von Zivilgesellschaft und Politik
in Zeiten des Web 2.0
Gisela ERLER, Staatsrätin für Zivilgesellschaft
und Bürgerbeteiligung des Landes Baden-Würt-
temberg, Stuttgart

Gemeinsame Tagung des Europa Zentrums BadenWürttemberg, der MFG Innovationsagentur für IT und Medien, Baden-Württemberg, des ArbeitskreiStiftung für Kommunikationsforschung

Medienpartner SWRinfo

$$
\text { 25.-26. Juni 2012, Stuttgart }
$$

Florian SETZEN, Direktor, Europa Zentrum Ba-

Ulrich WINCHENBACH, Leiter Projektteam Weiterbildung/Events, MFG Innovationsagentur für IT jekte/Business Development/Communications, MFG Innovationsagentur für IT und Medien BaWürttemberg, Stuttgart

Florian H. SETZEN, Direktor, Europa Zentrum BaDas Verhältnis von Zivilgesellschaft und Politik in Zeiten des Web 2.0

und Bürgerbeteiligung des Landes Baden-Würt-

* Julian Plottka, Wissenschaftlicher Mitarbeiter am Institut für Europäische Politik, Berlin.

1 Die einzelnen Beiträge zur Tagung sind dokumentiert in Florian H. Setzen (Hrsg.): Europapolitische Kommunikation und Web 2.0 - Formen, Trends, Herausforderungen und Chancen, Europapolitische Schriften des Europa Zentrums Baden-Württemberg, Band 4, Stuttgart 2012. 
Gegenständen der Tagung. Der Prozess der europäischen Integration schreitet weiter voran und verändert somit den Rahmen europapolitischer Kommunikation. Auch der Einsatz sozialer Medien in der politischen Kommunikation ist kontinuierlicher Veränderung unterworfen. Diese ergibt sich durch den technischen Fortschritt, der immer neue Instrumente im Bereich sozialer Medien hervorbringt, genauso wie durch Veränderungen in deren Nutzung durch Politiker und Bürger. Umso wichtiger ist es, dass sich die Wissenschaft ernsthaft mit der Bedeutung der sozialen Medien für die politische Kommunikation und ihrer Wirkung auf die repräsentative Demokratie auseinandersetzt. Dies gilt nicht nur aber auch für die Europapolitik als Forschungsgegenstand der Politikwissenschaft.

\section{Was sind soziale Medien und welche Nutzertypen gibt es?}

Eine Einführung in die Welt des Web 2.0 an der Schnittstelle von technischer Entwicklung und Nutzungsverhalten gaben Evandro Oliveira und Ulrich Winchenbach. Sie charakterisierten den qualitativen Unterschied zwischen sozialen und traditionellen Medien dadurch, dass die Technik dem Einzelnen aus einer reinen Rezipientenrolle heraus die Möglichkeit gebe in den sozialen Medien Produzent zu werden. Dazu stünde den Nutzern eine Vielzahl unterschiedlicher Medien (Video-Dateien, Fotos, etc.) zur Verfügung, die sich bei spezialisierten Anbietern (zum Beispiel YouTube für Videos) speichern und von dort direkt in den eigenen Blog oder die eigene Homepage einbinden ließen. Links zu den so bereitgestellten Informationen ließen sich dann über soziale Medien wie Twitter, Facebook, Google+ etc. aber auch per E-Mail verbreiten.

Dieser Wandel sei von einer Steigerung der Reichweite individueller Kommunikation begleitet, die noch weiter voranschreite, und mit einem steigenden Informationsniveau einhergehe: Während es 2011 129,8 Millionen Internetnutzer in Westeuropa gegeben habe, werde bis 2014 ein Anstieg auf 170,8 Millionen erwartet.
„Parlamentarier unter ,Druck“" - Wie bestimmen Facebook, Twitter und Co. den Politikeralltag im Europäischen Parlament?

Rainer WIELAND, MdEP, Vizepräsident des Europäischen Parlaments/Präsident der Europa-Union Deutschland, Stuttgart/Brüssel/Berlin

Heide RÜHLE, MdEP, Stuttgart/Brüssel

Michael THEURER, MdEP, Horb/Brüssel

Social Media - Relevante Plattformen, Anwendungsmöglichkeiten und Potential

Evandro OLIVEIRA und Ulrich WINCHEN$\mathrm{BACH}, \mathrm{MFG}$ Innovationsagentur für IT und Medien Baden-Württemberg, Stuttgart

Die Umsetzung der neuen Europäischen Bürgerinitiative - eine Zwischenbeurteilung Prof. Dr. Robert MÜLLER-TÖRÖK, Hochschule für öffentliche Verwaltung und Finanzen Ludwigsburg

Die Nutzung des web 2.0 durch die EUInstitutionen zur Kommunikation mit Bürgerinnen und Bürgern - Trends, Szenarien und Auswirkungen

Robert ANDRECS, Leiter des Referats für die Europa-Internetseite, Generaldirektion Kommunikation, Europäische Kommission, Brüssel

Podiumsdiskussion: Social Media in der europapolitischen Kommunikation - Hype oder Revolution? Wege zu mehr Bürgerbeteiligung in Europa mit Social Media

Peter FRIEDRICH, MdL, Minister für Bundesrat, Europa und Internationale Angelegenheiten in Baden-Württemberg, Stuttgart

Gunter CZISCH, Erster Bürgermeister der Stadt Ulm/Direktor des Donaubüros Ulm/Neu-Ulm

Robert ANDRECS, Leiter des Referats für die Europa-Internetseite, Generaldirektion Kommunikation, Europäische Kommission, Brüssel

Ronny PATZ, „Euro-Blogger“ mit dem Blog Polscieu auf der Plattform ,Ideas on Europe“, Potsdam Moderation: Arthur LANDWEHR, SWR-Chefredakteur Hörfunk, Stuttgart

Erfolg und Potential von Liquid Democracy

Alvar C. H. FREUDE, Diplom-KommunikationsDesigner, freiberuflicher Software-Entwickler, Autor, Berater und Trainer, Kuratoriumsmitglied bei Liquid democracy e.V., als Sachverständiger Mitglied in der Enquête-Kommission Internet und digitale Gesellschaft des Deutschen Bundestags, Stuttgart 
Jedoch bedienten sich nicht alle Nutzer des Internets in gleicherweise sozialer Medien. Eine Typologie von Forrester Research aufgreifend unterschieden Oliveira und Winchenbach sieben Typen von Nutzern sozialer Medien: Inaktive, Zuschauer, Mitglieder, Informationssammler, Kritiker, Plauderer und Schöpfer. Entsprechend vollziehe sich auch beim einzelnen User die Entwicklung des individuellen Nutzungsverhaltens von Ad-hoc-Versuchen und experimenteller Nutzung über die Integration sozialer Medien in die eigene Kommunikationsstrategie bis hin zur Veränderung des eigenen Kommunikationsverhaltens durch Anpassung an die sozialen Medien. Allen neuen Nutzern sozialer Medien empfahlen Oliveira und Winchenbach nach einer Phase der Beobachtung, eine Strategie zu entwickeln, mit welchen Zielen soziale Medien in die eigene Kommunikation integriert und welche Plattformen dafür genutzt werden sollen.

\section{Risiken und Chancen sozialer Medien: veränderte Kommunikation?}

Wie bereits der Überblick von Oliveira und Winchenbach andeutete, waren sich alle Referenten der Tagung einig, dass sich Kommunikation in den sozialen Medien sowohl von etablierten Medien als auch von direkter Kommunikation unterscheidet. Ob soziale Medien jedoch die individuelle Kommunikation verändern, hänge von deren Nutzung durch den Einzelnen ab. Die Referenten hoben dabei, entsprechend ihrem persönlichen Erfahrungshorizont, unterschiedliche Aspekte der Neuartigkeit von Kommunikation in den sozialen Medien hervor, die entweder als Chance oder auch als Risiko begriffen werden können.

Soziale Medien beschleunigten Kommunikation, bis hin zum Austausch oder der Verbreitung von Informationen in Echtzeit. Dass dies eine größere Verantwortung des einzelnen Nutzers erforderlich macht, darauf wies Peter Friedrich hin. In den sozialen Netzwerken ver-

\author{
Webbasierte Netzwerkstrukturen und ihre \\ demokratische Legitimation - Hilfe oder \\ Herausforderung für die repräsentative \\ Demokratie? \\ Dr. Annegret EPPLER, Politikwissenschaftlerin, \\ Universität Tübingen, und Dr. Henrik SCHELLER, \\ Politikwissenschaftler, Universität Potsdam \\ Die Welt des Web 2.0: Innovationen für die \\ formale und non-formale europapolitische \\ Bildung? - Tools, Nutzbarmachung, \\ Erfahrungen \\ Karsten LUCKE und Anselm SELLEN, Studien- \\ leiter, Europahaus Marienberg \\ Zusammenfassung und Gesamtschau auf die \\ Ergebnisse der Beiträge \\ Ulrich WINCHENBACH, MFG Innovationsagen- \\ tur für IT und Medien Baden-Württemberg, Stutt- \\ gart
}

liere Kommunikation ihren situativen Kontext, sodass leicht Missverständnisse entstünden. Dies gilt besonders, wenn die Reaktionszeiten immer kürzer werden. Michael Theurer zeigte zudem am Beispiel der in den sozialen Netzwerken geführten Diskussion über die Entscheidung des Europäischen Parlaments zum ACTA-Abkommen, ${ }^{2}$ dass Beschleunigung nicht zugleich Aktualität der verbreiteten Informationen bedeute. Diese seien in der Diskussion zum ACTA-Abkommen oftmals überholt gewesen. Auch Robert Andrecs stellte dar, dass eine der zentralen Aufgaben seines Referats in der Generaldirektion Kommunikation darin bestanden habe, Anfragen zum ACTAAbkommen systematisch auszuwerten und korrekte Informationen von Seiten der Europäischen Kommission möglichst in Echtzeit zur Verfügung zu stellen.

Als eine weitere qualitative Neuerung, die eine große Chance für die politische Kommunikation über soziale Medien darstellt, wurde die Reichweite der Kommunikation angesprochen. Diese sei einerseits in der geografischen

2 Legislative Entschließung des Europäischen Parlaments vom 4. Juli 2012 zu dem Entwurf eines Beschlusses des Rates über den Abschluss des Handelsübereinkommens zur Bekämpfung von Produkt- und Markenpiraterie, A7-0204/2012. 
Dimension erweitert: Gunter Czisch berichtete von den Erfahrungen des Donaubüros Ulm/ Neu-Ulm mit dem Einsatz sozialer Medien, um ehrenamtlich Aktive entlang der gesamten Donau dauerhaft in ein Netzwerk einzubinden. Dies sei allein schon aus finanziellen Gründen nur über soziale Medien und das Internet möglich. Andererseits sei die Reichweite aber auch in der sozialen Dimension erweitert: So gebe es inzwischen Bürger, die nur noch über soziale Medien für politische Kommunikation erreichbar sind. Laut Andrecs hat die Europäische Kommission insbesondere Jüngere, Selbständige und Einwohner ländlicher Regionen als ,Problemgruppen' identifiziert, die zwar Interesse an europäischer Politik hätten, sich aber nicht ausreichend informiert fühlten. $G i$ sela Erler erinnerte aber auch daran, dass weiterhin große Teile der Bevölkerung die sozialen Medien nicht nutzen und eine Umstellung politischer Kommunikation nicht zu deren Exklusion aus politischen Debatten führen dürfe.

Ein Beispiel für die Exklusivität von europapolitischer Kommunikation in den sozialen Medien lieferte der Euro-Blogger Ronny Patz, dessen Blog zu aktuellen europapolitischen Themen sich primär an eine Fachöffentlichkeit richtet. Erst wenn Journalisten seine Beiträge aufgriffen, was gelegentlich geschehe, erreichten sie eine breitere Öffentlichkeit. Damit ist aber gleichzeitig ein weiterer Unterschied zu den klassischen Medien angesprochen: Mit dem Wandel vom Rezipienten zum Produzenten erhält der Nutzer zumindest eine potenzielle Agenda-Setzungsmacht. Dass diese oftmals noch bei den klassischen Massenmedien liege, illustrierte Theurer anhand der Diskussion über das ACTA-Abkommen. Dieses sei in den sozialen Medien erst thematisiert worden, nachdem sich die klassischen Massenmedien damit befasst hätten.

Als eine weitere Veränderung, die sich aus der Bi-Direktionalität der Kommunikation in den sozialen Medien ergibt, wurde die Dialogorientierung der sozialen Medien hervorgehoben.
Die strenge Trennung der Sender- und Empfängerrollen sei aufgehoben, was ein großes Potenzial für die Nutzung sozialer Medien zur Stärkung politischer Partizipation biete. Als aktuelle Beispiele wurden die von Friedrich verantworteten Projekte der baden-württembergischen Landesregierung zur Einbindung von Bürgern in den Prozess der Strategieformulierung für die Entwicklungshilfe- und Europapolitik sowie die Begleitung der Diskussion über das Projekt, citybahnhof.ulm‘ im Internet, ${ }^{3}$ die Czisch vorstellte, genannt.

Die bereits angesprochene Kostensenkung ist ein weiterer Unterschied zu traditionellen Medien, deren Produktion wesentlich teurer ist. Ein anschauliches Beispiel für die Kostensenkung war die Bandbreite digitaler Instrumente derer sich allein die anwesenden Mitglieder des Europäischen Parlaments, Michael Theurer, Heide Rühle und Rainer Wieland, für ihre Kommunikation bedienen: Diese reicht klassisch von E-Mails, der eigenen Homepage und dem eigenen Newsletter, über bekannte Portale wie Facebook, Xing, Flickr und dem Kurznachrichtendienst Twitter bis hin zur eigenen App von Wieland.

Mit der Senkung der Kosten für Kommunikation gehe auch die Gefahr des ,information overflow' einher. So überschreite die Zahl der E-Mails, die bei den genannten Mitgliedern des Europäischen Parlaments täglich eingehen, zum Teil die Marke der 1.000. Insbesondere Standard-Massen-E-Mails seien eher ein Problem denn eine Form ernsthafter politischer Partizipation.

Während die bisher genannten Unterschiede von den Teilnehmern trotz möglicher Risiken als Vorteile der Kommunikation in den sozialen Medien begrüßt wurden, war bei zwei weiteren Unterschieden umstritten, ob sie ein ernsthaftes Risiko politischer Kommunikation in den sozialen Medien darstellen. Insbesondere Wieland thematisierte das bisherige Fehlen allgemein akzeptierter Standards für den

3 Vgl. http://www.citybahnhof.ulm.de/ (letzter Zugriff: 13.7.2012). 
Umgang auf sozialen Netzwerken. Theurer stimmte dem zu, identifizierte jedoch eine generelle Tendenz zur „Parlamentsbeschimpfung", die nicht allein auf das Verhalten der Bürger gegenüber Abgeordneten im Web 2.0 begrenzt sei.

In engem Zusammenhang mit der Frage nach der ,Netikette' in den sozialen Medien steht jene nach der Anonymität im Internet, die im Spannungsverhältnis von Datenschutz und Verantwortlichkeit zu diskutieren ist. Während Wieland bekannte, nur auf Anfragen von Personen zu antworten, die auch identifizierbar sind, berichtete Alvar C. H. Freude aus der Arbeit der Enquete-Kommission „Internet und digitale Gesellschaft" des Deutschen Bundestages, dass nach langer Diskussion über Verifizierungsmechanismen in der die EnqueteKommission begleitenden Online-Debatte auch Beiträge unter Pseudonym akzeptiert würden. Der Unterschied zur direkten Interaktion der Bürger mit ihren Vertretern bestehe jedoch darin, dass die Enquete-Kommission als Filter fungiere bevor die Ergebnisse den Abgeordneten vorgelegt würden, während der einzelne Abgeordnete oft nicht entscheiden kann, ob es sich um echte Bürger oder gefälschte Identitäten handelt. Dieses Problem sah auch Müller-Török als eine der zentralen Gefahren für das Instrument der Europäischen Bürgerinitiative, da seiner Ansicht nach die Verifizierungsmechanismen im Anschluss an die Sammlung der Unterstützungsbekundungen für eine Bürgerinitiative unzureichend seien.

Weiterhin war die Frage umstritten, ob die stärkere Emotionalisierung der Kommunikation in den sozialen Medien ein Vor- oder ein Nachteil sei. Rühle legte einerseits dar, dass sich die Debatten in den sozialen Medien zum Teil auf dem Niveau der Boulevard-Presse bewegten und sich bestimmte Themen nicht mit , 140 Zeichen', der maximalen Länge eines Tweets, vermitteln ließen. Newsletter oder die eigene Homepage ermöglichten dagegen eine differenziertere Darstellung von Themen. Andrecs wies dagegen darauf hin, dass die sozialen Me- dien aus Sicht der Europäischen Kommission eine Chance seien, ihre oftmals sehr textlastige Kommunikation populärer zu gestalten. Auch Friedrich sah in einer Emotionalisierung politischer Debatten keinen generellen Nachteil, sondern auch eine Chance.

\section{Soziale Medien: Herausforderung für Politiker und Bürger?}

In Abhängigkeit von der jeweiligen Nutzungsintensität der sozialen Medien durch den Einzelnen stellen diese sowohl Bürger als auch Politiker vor neue Herausforderungen. Die Vielzahl der zur Verfügung stehenden Formate, Medien, Plattformen und Instrumente macht individuelles Lernen im Bereich der Technik bereits für den passiven Gebrauch sozialer Medien als Rezipient unabdingbar. Auch die rasante Zunahme an bereitgestellten Informationen stellt neue Anforderungen an die Informationsverarbeitung durch die Bürger, abgesehen davon, dass, wie von Andrecs und Theurer angedeutet, die Qualität der Informationen im Einzelfall vom Nutzer zu hinterfragen ist.

Wie solche Lernprozesse in der europapolitischen Bildungsarbeit konkret gestaltet werden können, darüber berichteten Karsten Lucke und Anselm Sellen aus ihrer Arbeit im EuropaHaus Marienberg. In den dort durchgeführten Seminaren werden Jugendlichen anhand der Bearbeitung europapolitischer Fragestellungen mithilfe sozialer Medien die Chancen und Risiken des Web 2.0 vermittelt. Ziel der Workshops sei es die Funktionslogiken der einzelnen Instrumente zu vermitteln und so die $\mathrm{Zu}$ gangsschwelle zu Online-Partizipation zu senken. Gleichzeitig würden die Jugendlichen zur inhaltlichen Reflexion über thematische Fragestellungen angeregt.

In den Seminaren würden die Teilnehmer in die Rolle von Produzenten versetzt, die unter Einsatz unterschiedlichster Medien zu jedem Seminar einen eigenen Blog gestalteten. Dass die Produzenten-Rolle mehr noch als jene des Rezipienten neue Herausforderungen stellt, 
machten bereits die Handlungsempfehlungen von Oliveira und Winchenbach deutlich. Mit Blick auf seine Erfahrungen aus dem Donaubüro Ulm/Neu-Ulm und dem Projekt, citybahnhof.ulm'stellte Czisch fest, dass für eine gute Kommunikation Experten notwendig seien. Dies ist auch Ziel des von Andrecs geleiteten Referats in der Generaldirektion Kommunikation der Europäischen Kommission: Einerseits solle es die gesamten Aktivitäten der Europäischen Kommission in den sozialen Medien koordinieren und andererseits einen Erfahrungsaustausch zwischen deren Mitarbeitern und Vertretern organisieren, um den Umgang mit den sozialen Medien zu professionalisieren.

Die Bedeutung der sozialen Medien sei jedoch inzwischen so groß, darin waren sich alle Referenten einig, dass ein Politiker heute auf deren Nutzung nicht mehr verzichten könne. In zunehmendem Maße stelle sich diese Frage aber auch für die Verwaltungen. Hier einen Bewusstseinswandel in den Amtsstuben herbeizuführen, sei eine der zentralen Herausforderungen. Dazu sei einerseits die Beratung und Schulung der Verwaltungen notwendig, andererseits gebe es aber auch noch viele ungeklärte Fragen, zum Beispiel ob es sich bei einem von einer Behörde versandten Tweet um einen Rechtsakt mit den entsprechenden juristischen Folgen handelt oder nicht. Solange solche Fragen nicht abschließend geklärt seien, erschwere dies den Einsatz sozialer Medien.

Als ein Beispiel für mangelnde Offenheit politischer Institutionen und ihrer Mitarbeiter gegenüber neuen Formen der politischen Partizipation stellte Robert Müller-Török die Europäische Bürgerinitiative dar. Diese sei prinzipiell ein begrüßenswertes Instrument, aber eine Reihe nicht abschließend geklärter Fragen drohe, sie de facto nutzlos zu machen. Als Probleme führte er Haftungsfragen, insbesondere im Bereich des Datenschutzes, die Ungleichbehandlung der Bürger unterschiedlicher EUMitgliedstaaten hinsichtlich ihrer Beteiligungsrechte sowie die extrem hohen technischen Anforderungen an die Systeme zur
Sammlung digitaler Unterstützungsbekundungen an.

\section{Soziale Medien: eine Bedrohung der repräsentativen Demokratie?}

Ob vergleichbare Probleme auch durch die Nutzung sozialer Medien für die repräsentative Demokratie insgesamt drohen, war die zentrale Frage der Tagung. In der Grundtendenz waren sich jedoch alle Teilnehmer bei der Beantwortung der Frage einig: Die sozialen Medien und neue digitale Instrumente zur Bürgerbeteiligung bedrohen die repräsentative Demokratie nicht. Statt von einer Revolution durch die neuen technischen Instrumente sei von einer Ergänzung der bestehenden politischen Institutionen auszugehen.

Annegret Eppler und Henrik Scheller betteten die Frage nach dem von den sozialen Medien und neuen Kommunikationsformen ausgelösten Wandel repräsentativer Demokratie in die Postparlamentarismusdebatte und die Diskussion über die generellen Herausforderungen ein, denen sich Demokratie heute gegenüber sieht. Sie hoben dabei insbesondere die Prozesse der Globalisierung, der Europäisierung und des demografischen Wandels, unter Einbeziehung der Rolle, die hier Migrationsbewegungen zukommt oder zukommen kann, hervor. Diese Entwicklungen führten zu einem Wandel der Rolle von Parlamenten in der repräsentativen Demokratie. Zwar gebe es eine generelle Tendenz der Verlagerung von Entscheidungskompetenzen auf die obersten Ebenen, dies ginge jedoch nicht mit einem Niedergang der Parlamente einher. Vielmehr müssten sich diese von der Wahrnehmung eigener Entscheidungskompetenzen hin zu einer besseren Wahrnehmung ihrer Artikulationsfunktion umorientieren. Angesichts zunehmender Entscheidungskomplexität sei die Einbindung einzelner Parlamente in fachpolitische Netzwerke eine sinnvolle Strategie.

Eppler und Scheller sprachen zwei weitere zentrale Entwicklungen an, die in einem engen Zusammenhang mit der Nutzung sozialer Me- 
dien für die (europa-)politische Kommunikation stehen: Dies waren jene der gesellschaftlichen Individualisierung sowie jene zunehmender Fragmentierung virtueller Diskursräume bei gleichzeitiger wirtschaftlicher Konzentration und Kommerzialisierung im Geschäftsfeld Internet.

Diese Entwicklungen stellten die repräsentative Demokratie vor konkrete Herausforderungen im Umgang mit politischer Kommunikation und Partizipation in sozialen Medien und im Internet. In der institutionellen Dimension seien folgende Fragen zu klären: Soll das Internet zur Entscheidungsfindung genutzt werden und/oder zur Sammlung von politischen Präferenzen dienen? Inwiefern sind letztere, die im Internet geäußert werden, als repräsentativ einzuschätzen? In der prozessualen Dimension sei zu beachten, dass die Debatte in den sozialen Medien für den politischen Diskurs insgesamt relevant sei und nicht nur die Bürger betreffe, die die sozialen Medien nutzten. Um Antworten auf diese Fragen zu finden, seien konkrete Herausforderungen zu bewältigen: Wie lässt sich Entscheidungsfindung im Internet organisieren? Wie lassen sich Netzdiskurse in den gesamtpolitischen Diskurs übertragen? Wie lässt sich Schwarmintelligenz als Ressource für sachpolitische Entscheidungen nutzen?

Als technisches Instrument, das versucht eine Antwort auf diese Fragen zu geben, stellte Freude die Plattform ,liquid democracy' vor, die neben, adhocracy' die am weitesten entwickelte Plattform darstellt. Jedoch seien prinzipiell viele weitere Anwendungen aus dem Bereich der sozialen Medien zur Organisation von politischen Debatten und von Entscheidungsfindung im Internet einsetzbar. Ziel der Entwicklung von, liquid democracy' sei es, ein gerechtes Wahlsystem zu schaffen, das auch Abstimmungen über Sachentscheidungen ermöglicht.

,Liquid democracy“ selbst als Programm werde von Liquid Democracy e.V. entwickelt. Es könne zur Organisation von politischen Debat- ten und zur Entscheidungsfindung in beliebigen Kontexten und für beliebige Personengruppen genutzt werden. So setze die PiratenPartei beispielsweise, liquid democracy‘ ein, um parteiinterne Debatten zu führen und Entscheidungen zu treffen, an denen nur Parteimitglieder teilnehmen dürfen. ,Liquid democracy“ ermögliche es jedem Nutzer, selbst Themen zu setzen und an Diskussionen und Abstimmungen über von anderen Nutzern gesetzte Themen teilzunehmen. Grundlage jeder Themensetzung sei ein Vorschlag für einen Text, an dem in der ersten Phase, der politischen Debatte, gemeinsam gearbeitet werde. Dieser Phase der gemeinsamen Textproduktion schließe sich die Abstimmung über einzelne Textteile an. Das Ende der Debatte sei die Abstimmung über den Gesamttext. Dabei könne jeder Nutzer entweder an jeder Abstimmung selbst teilnehmen oder aber seine Stimme delegieren. Die Idee hinter der Delegation sei mit der Funktionslogik von Arbeitsparlamenten vergleichbar. Jeder Nutzer solle sich auf die Arbeit an Themen, die ihn am meisten interessieren, konzentrieren und für alle anderen Themen seine Stimme delegieren.

Inzwischen funktioniere, liquid democracy', dennoch gebe es zentrale Herausforderungen, die noch zu bewältigen seien. So reduziere die Software keine Entscheidungskomplexität, sondern sei nur ein alternatives Entscheidungsverfahren. Bei Schlussabstimmungen sei die Beteiligung in der Regel sehr gering. Zudem müssten noch Verfahren zum Umgang mit sogenannten Trollen, also Nutzern, die das System bewusst stören, und gefälschten Identitäten gefunden werden. Technisch sei beides realisierbar, es sei jedoch zwischen den Zielen einer effizienten Entscheidungsfindung und der Bewahrung der Freiheit im Internet sowie des Datenschutzes abzuwägen. Auch sei das Delegationssystem noch zu verbessern, indem Delegationen nur noch zeitlich befristet vergeben werden können. So wird vermieden, dass einzelne Nutzer delegierte Stimmen längst ausgeschiedener Nutzer auf Dauer behalten. Ferner sei die Responsivität der Delegierten gegen- 
über den Deligierenden noch nicht sehr weit entwickelt.

Zentrale Herausforderung ist jedoch die Verknüpfung digitaler Entscheidungsfindungssysteme mit bestehenden politischen Systemen. Aufgrund der Größe moderner demokratischer Systeme, ließen sich, so die einhellige Meinung der Diskutanten, ,liquid democracy“ und vergleichbare Plattformen nicht für alle Bürger eines Staates umsetzen. In diesem Sinne äußerte Patz die Hoffnung, dass sich politische Kommunikation mittels sozialer Medien nicht $\mathrm{zu}$,,liquid democracy“, sondern [zu] listening democracy" entwickele.

Wie von Czisch und Andrecs angemerkt wurde, bieten die sozialen Medien als Sensorium der Politiker die Möglichkeit, die Inklusivität bestehender politischer Systeme zu erhöhen. Politiker müssten jedoch lernen zu differenzieren, wann es sich um einen, Sturm im digitalen Wasserglas ' und wann um die ernsthafte Äußerung von politischen Anliegen handelt.

Grundsätzlich sei die Entscheidung durch Repräsentanten weiterhin gewünscht, auch wenn sich diese an die neuen Kontextbedingungen der Politik anpassen müssten. Einige dieser veränderten Kontextbedingungen des Handelns der Politiker wurden auf der Tagung an konkreten Beispielen aufgezeigt.

Friedrich hob angesichts seiner Erfahrungen aus Bürgersprechstunden hervor, dass Bürger mit ihrem Fachwissen inzwischen Spezialisten ihrer eigenen Anliegen seien, und wies daraufhin, dass es zunehmend Aufgabe von Politikern sei, Ausgleichsprozesse zwischen Spezialinteressen zu organisieren. Dies bedeute einen Verlust an Kontrolle über die politische Agenda. Auch verlören die örtlichen Gliederungen der Parteien ihre intermediäre Rolle zugunsten direkter Interaktion von Bürgern und Politikern.

Patz deutete die Mobilisierung gegen das ACTA-Abkommen im Internet als eine erstmalige Politisierung europäischer Entscheidungsprozesse, auf die die Unionsinstitutionen nicht vorbereitet gewesen seien. Friedrich stimmte der Analyse einer mangelnden Politisierung auf Unionsebene durch das Fehlen des Zusammenspiels von Regierungsmehrheit und Opposition zu, sah aber in den Diskussionen über den Verfassungsvertrag und die Dienstleistungsrichtlinie ${ }^{4}$ vergleichbare Beispiele für eine Politisierung der europapolitischen Diskussion.

Während die von Friedrich geschilderten Erfahrungen und das Beispiel des ACTA-Abkommens Ausdruck neugewachsener Teilhabeerwartungen der Bürger sind, zeigte Czisch am Beispiel der Debatten über das Projekt, citybahnhof.ulm', dass Politik sich auch der sozialen Medien bedienen kann, um zur Partizipation zu motivieren. Zur Vorbereitung des Diskussions- und Entscheidungsprozesses sind die Planungsideen zum Umbau des Ulmer Hauptbahnhofes im Internet zugänglich gemacht worden. Zwar seien auch diese Informationen zuerst durch eine Fachöffentlichkeit diskutiert worden, aber die digitale Bereitstellung senke die Zugangsschwelle auch für fachfremde Bürger. In dem Projekt werden online und offline Kanäle direkt verknüpft mit dem Ziel, Bürger durch das Internet zur Teilnahme an lokalen Foren zu gewinnen. In diesem Beispiel fungiert das Internet als Eintrittstor zur politischen Teilhabe.

4 Richtlinie 2006/123/EG des Europäischen Parlaments und des Rates vom 12. Dezember 2006 über Dienstleistungen im Binnenmarkt, in: Amtsblatt der EU, Nr. L 376 vom 27. Dezember 2006, S. 36-68. 